\begin{tabular}{|c|l|}
\hline Title & Thermopower modulation clarification of the intrinsic effective mass in transparent oxide semiconductor BaSnO3 \\
\hline Author(s) & Sanchela, A nup V.; Onozato, Takaki; Feng, Bin; Ikuhara, Y uichi; Ohta, Hiromichi \\
\hline Citation & $\begin{array}{l}\text { Physical Review Materials, 1(3), 34603 } \\
\text { https://doi.org/10.1103/PhysRevMaterials.1.034603 }\end{array}$ \\
\hline Issue Date & 2017-08-04 \\
\hline Doc URL & http://hdl.handle.net/2115/68299 \\
\hline Rights & ○ 2017 A merican Physical Society \\
\hline Type & article \\
\hline File Information & PhysRevMaterials.1.034603.pdf \\
\hline
\end{tabular}

Instructions for use 


\title{
Thermopower modulation clarification of the intrinsic effective mass in transparent oxide semiconductor $\mathrm{BaSnO}_{3}$
}

\author{
Anup V. Sanchela, ${ }^{1, *}$ Takaki Onozato, ${ }^{2}$ Bin Feng, ${ }^{3}$ Yuichi Ikuhara, ${ }^{3}$ and Hiromichi Ohta ${ }^{1, \dagger}$ \\ ${ }^{1}$ Research Institute for Electronic Science, Hokkaido University, N20W10, Kita, Sapporo 001-0020, Japan \\ ${ }^{2}$ Graduate School of Information Science and Technology, Hokkaido University, N14W9, Kita, Sapporo 060-0814, Japan \\ ${ }^{3}$ Institute of Engineering Innovation, The University of Tokyo, 2-11-16 Yayoi, Bunkyo, Tokyo 113-8656, Japan
}

(Received 5 April 2017; revised manuscript received 12 June 2017; published 4 August 2017)

\begin{abstract}
The exact intrinsic carrier effective mass $m^{*}$ of a well-studied transparent oxide semiconductor $\mathrm{BaSnO}_{3}$ is unknown because the reported $m^{*}$ values are scattered from $0.06 m_{0}$ to $3.7 m_{0}$. This paper identifies the intrinsic $m^{*}$ of $\mathrm{BaSnO}_{3}, m^{*}=0.40 \pm 0.01 m_{0}$, by the thermopower modulation clarification method and determines the threshold of the degenerate/nondegenerate semiconductor. At the threshold, the thermopower values of both the La-doped $\mathrm{BaSnO}_{3}$ and $\mathrm{BaSnO}_{3}$ thin-film transistor structures are $240 \mu \mathrm{V} \mathrm{K}^{-1}$, the bulk carrier concentration is $1.4 \times 10^{19} \mathrm{~cm}^{-3}$, and the two-dimensional sheet carrier concentration is $1.8 \times 10^{12} \mathrm{~cm}^{-2}$. When the Fermi energy $E_{\mathrm{F}}$ is located above the parabolic shaped conduction band bottom, the mobility is rather high. In contrast, $E_{\mathrm{F}}$ below the threshold exhibits a very low carrier mobility, most likely because the tail states suppress the carrier mobility. The present results are useful to further develop $\mathrm{BaSnO}_{3}$-based oxide electronics.
\end{abstract}

DOI: 10.1103/PhysRevMaterials.1.034603

Transparent oxide semiconductors (TOSs) with a relatively high electrical conductivity and a large band gap $\left(E_{g}>\right.$ $3.1 \mathrm{eV}$ ) are commonly used as transparent electrodes and channel semiconductors for thin-film transistor (TFT) driven flat panel displays (FPDs) such as liquid crystal displays (LCDs) and organic light emitting diodes (OLEDs) [1]. TOS materials include $\mathrm{Sn}$-doped $\mathrm{In}_{2} \mathrm{O}_{3}$ (ITO) and $\mathrm{InGaZnO}_{4}$-based oxides. Novel TOSs exhibiting higher carrier mobilities have been intensively explored since the TFT performance strongly depends on the carrier mobility of the channel semiconductor. In 2012, Kim et al. reported that a La-doped $\mathrm{BaSnO}_{3}$ (space group $P m \overline{3} m$, cubic perovskite structure, $a=4.115 \AA$, $E_{\mathrm{g}} \sim$ $3.1 \mathrm{eV}$ ) single crystal grown by the flux method exhibits a very high mobility $\left(\mu_{\text {Hall }} \sim 320 \mathrm{~cm}^{2} \mathrm{~V}^{-1} \mathrm{~s}^{-1}\right)$ at room temperature $[2,3]$. This report inspired the current interest in $\mathrm{BaSnO}_{3}$ films and $\mathrm{BaSnO}_{3}$-based TFTs [4-9].

Since the mobility is expressed as $\mu=e \tau m^{*-1}$, where $e$, $\tau$, and $m^{*}$ are the electron charge, carrier relaxation time, and carrier effective mass, respectively, the high mobility of the Ladoped $\mathrm{BaSnO}_{3}$ single crystal should be due to both a small $m^{*}$ and a large $\tau$. Generally, the $\tau$ value of epitaxial films is smaller than that of the bulk single crystal due to the fact that the carrier electrons are scattered at dislocations, which originated from the lattice mismatch $(\delta)$ and at other structural defects, in addition to optical phonon scattering. The estimated misfit dislocation spacing $d$ is $7.4 \mathrm{~nm}$ because $\delta$ between $\mathrm{BaSnO}_{3}$ and $\mathrm{SrTiO}_{3}(a=3.905 \AA)$ is $+5.3 \%$. $\mathrm{BaSnO}_{3}$ films grown on $(001)$ $\mathrm{SrTiO}_{3}$ substrates exhibit rather small mobilities $\left(\mu_{\text {Hall }} \sim\right.$ $\left.26-100 \mathrm{~cm}^{2} \mathrm{~V}^{-1} \mathrm{~s}^{-1}\right)[3,7,10]$ compared with those grown on (001) $\mathrm{PrScO}_{3}(a=4.026 \AA, \delta=+2.2 \%, d \sim 17.7 \mathrm{~nm})$ $\left(\mu_{\text {Hall }} \sim 150 \mathrm{~cm}^{2} \mathrm{~V}^{-1} \mathrm{~s}^{-1}\right)$ [7]. On the other hand, $m^{*}$ only depends on the electronic structure of the material. Many theoretical and experimental values of $m^{*}$, which were mostly determined from the optical properties, have been reported (e.g., theoretical values of $\sim 0.06 m_{0}$ [11], $\sim 0.4 m_{0}$ [3], and

\footnotetext{
*anup.sanchela@es.hokudai.ac.jp

†hiromichi.ohta@es.hokudai.ac.jp
}

$\sim 0.2 m_{0}$ [12], and experimental values of $3.7 m_{0}$ [13], $0.61 m_{0}$ [14], $\sim 0.35 m_{0}$ [15], $\sim 0.396 m_{0}$ [16], $0.27 \pm 0.05 m_{0}$ [10], $\left.0.19 \pm 0.01 m_{0}[8]\right)$. Consequently, determining the intrinsic $m^{*}$ value is almost impossible.

We hypothesized that the experimental values include errors since the substrate contributes to the optical spectra of the $\mathrm{BaSnO}_{3}$ films. To overcome this difficulty, we use the thermopower $S$ modulation clarification method to determine the intrinsic $m^{*}$ of $\mathrm{BaSnO}_{3}$ as $S$ clearly reflects the energy derivative of the density of states (DOS) at the Fermi energy $E_{\mathrm{F}}$. This study measures the $S$ of La-doped $\mathrm{BaSnO}_{3}$ films and $\mathrm{BaSnO}_{3}$ with a TFT structure as functions of carrier concentration. The carrier concentration can be modulated by applying an electric field. The intrinsic $m^{*}=0.4 \pm 0.01 m_{0}$ is clarified when $E_{\mathrm{F}}$ is located above the degenerate/nondegenerate threshold of $\mathrm{BaSnO}_{3}$.

La-doped $\mathrm{BaSnO}_{3}$ films (thickness $\sim 200 \mathrm{~nm}$ ) were heteroepitaxially grown on (001) $\mathrm{SrTiO}_{3}$ single crystal substrates by pulsed laser deposition (PLD, KrF excimer laser, $\lambda=$ $248 \mathrm{~nm}, 10 \mathrm{~Hz}$, fluence $\sim 2 \mathrm{~J} \mathrm{~cm}^{-2}$ pulse $^{-1}$ ) technique using dense ceramic disks of $\mathrm{Ba}_{1-x} \mathrm{La}_{x} \mathrm{SnO}_{3}(0.001 \leqslant x \leqslant 0.07)$ as the targets. The substrate temperature and oxygen pressure during film growth were $700^{\circ} \mathrm{C}$ and $10 \mathrm{~Pa}$, respectively. Since the surface of the as-deposited $\mathrm{BaSnO}_{3}$ films was composed of tiny $(\sim 50 \mathrm{~nm})$ grains, the film was annealed at $1200^{\circ} \mathrm{C}$ in air to obtain atomically smooth surfaces [17]. High-resolution x-ray diffraction ( $\mathrm{Cu} K \alpha_{1}$, ATX-G, Rigaku) measurements revealed that the resultant films were heteroepitaxially grown on (001) $\mathrm{SrTiO}_{3}$ substrates with a cube-on-cube epitaxial relationship (see Fig. S2 in the Supplemental Material [18]). The film thickness of the resultant films was calculated from the Pendellösung fringes, which were observed around the 002 diffraction peak of La-doped $\mathrm{BaSnO}_{3}$. The topographic atomic force microscopy (AFM) image of the resultant films showed a stepped and terraced surface.

We then measured the electrical conductivity $\sigma$, carrier concentration $n$, and Hall mobility $\mu_{\text {Hall }}$ of the La-doped $\mathrm{BaSnO}_{3}$ films at room temperature by the conventional dc four-probe method using an In-Ga alloy electrode with van 
TABLE I. Electrical properties of La-doped $\mathrm{BaSnO}_{3}$ films at room temperature.

\begin{tabular}{lcccc}
\hline \hline$x$ in $\mathrm{Ba}_{1-x} \mathrm{La}_{x} \mathrm{SnO}_{3}$ & $\sigma\left(\mathrm{S} \mathrm{cm}^{-1}\right)$ & $n\left(\mathrm{~cm}^{-3}\right)$ & $\mu_{\text {Hall }}\left(\mathrm{cm}^{2} \mathrm{~V}^{-1} \mathrm{~s}^{-1}\right)$ & $S(\mu \mathrm{V} \mathrm{K})$ \\
\hline 0.001 & 0.00481 & $2.10 \times 10^{18}$ & 0.0143 & -253 \\
0.004 & 1.32 & $8.80 \times 10^{18}$ & 0.935 & -243 \\
0.0055 & 66.4 & $3.24 \times 10^{19}$ & 3.8 & -188 \\
0.007 & 420 & $7.75 \times 10^{19}$ & 33.8 & -126 \\
0.02 & 2620 & $2.66 \times 10^{20}$ & 67.6 & -57.2 \\
0.05 & 6910 & $6.41 \times 10^{20}$ & 43.9 & -31.3 \\
0.07 & 3350 & $4.76 \times 10^{20}$ & 4.4 \\
\hline \hline
\end{tabular}

der Pauw geometry. $S$ was measured at room temperature by creating a temperature gradient $\Delta T$ of $\sim 4 \mathrm{~K}$ across the film using two Peltier devices (and two small thermocouples were used to monitor the actual temperatures of each end of the $\mathrm{Ba}_{1-x} \mathrm{La}_{x} \mathrm{SnO}_{3}$ films). The thermoelectromotive forces $\Delta V$ and $\Delta T$ were measured simultaneously. The $S$ values were obtained from the slope of the $\Delta V-\Delta T$ plots.

Table I summarizes the electrical properties of the Ladoped $\mathrm{BaSnO}_{3}$ films at room temperature. $\sigma$ rapidly increased with $x$ in $\mathrm{Ba}_{1-x} \mathrm{La}_{x} \mathrm{SnO}_{3}$ and reached a maximum value of $6910 \mathrm{~S} \mathrm{~cm}^{-1}$ in the $x=0.05$ sample. At the same time, $n$ and $\mu_{\text {Hall }}$ achieved the highest values of $6.41 \times 10^{20} \mathrm{~cm}^{-3}$ and $67.3 \mathrm{~cm}^{2} \mathrm{~V}^{-1} \mathrm{~s}^{-1}$, respectively. It should be noted that the $\mu_{\text {Hall }}$ value increased with increasing $n$, which is consistent with the data reported by Kim et al. [2]. The sign of $S$ was negative in all samples, which agreed with the fact that La-doped $\mathrm{BaSnO}_{3}$ is an $n$-type semiconductor. The absolute value $|S|$ gradually decreased with $n$.

To clarify the intrinsic $m^{*}$, we plotted the relationship between $n$ and $S$. As outlined in Eqs. (1)-(3), the DOS effective mass $m^{*}$ was calculated. When $n$ exceeded $\sim 10^{19} \mathrm{~cm}^{-3},|S|$ monotonically decreased with increasing $n$ (Fig. 1). We then calculated $m^{*}$ using the following relation between $n$ and $S$ [19],

$$
S=-\frac{k_{B}}{e}\left(\frac{(r+2) F_{r+1}(\xi)}{(r+1) F_{r}(\xi)}-\xi\right),
$$

where $k_{B}, \xi, r$, and $F_{r}$ are the Boltzmann constant, chemical potential, scattering parameter of relaxation time, and Fermi integral, respectively. $F_{r}$ is expressed as

$$
F_{r}(\xi)=\int_{0}^{\infty} \frac{x^{r}}{1+e^{x-\xi}} d x,
$$

and $n$ is given by

$$
n_{-}=4 \pi\left(\frac{2 m^{*} k_{B} T}{h^{2}}\right)^{3 / 2} F_{1 / 2}(\xi),
$$

where $h$ and $T$ are Planck's constant and the absolute temperature, respectively. We used an $r$ value of 0.5 , which indicates the carrier electrons are dominantly scattered by the optical phonon. Using Eqs. (1)-(3), we obtained the degenerate/nondegenerate threshold of $1.4 \times 10^{19} \mathrm{~cm}^{-3}$. The intrinsic effective mass was obtained as $m^{*}=0.40 \pm 0.01 m_{0}$ when $n \geqslant 1.4 \times 10^{19} \mathrm{~cm}^{-3}$, though the $S$ - $n$ plots at $n<1.4 \times$ $10^{19} \mathrm{~cm}^{-3}$ did not obey the $m^{*}=0.4 m_{0}$ line. The threshold of the degenerate/nondegenerate semiconductor appeared around $n=1.4 \times 10^{19} \mathrm{~cm}^{-3}$. As shown in the inset, when $n$ exceeded the threshold (三degenerate semiconductor, Fig. S1 [18]), $E_{\mathrm{F}}$ was located inside of the parabolic shaped conduction band. On the other hand, when $n$ was less than the threshold (三nondegenerate semiconductor), $E_{\mathrm{F}}$ was located at the nonparabolic shaped extra DOS. At the threshold, the $S$ value should be $240 \mu \mathrm{V} \mathrm{K}^{-1}$.

To further clarify the intrinsic $m^{*}$ of $\mathrm{BaSnO}_{3}$, we measured the electric field modulated $S$ of $\mathrm{BaSnO}_{3}$-based TFT (Fig. 2). First, a 22-nm-thick undoped $\mathrm{BaSnO}_{3}$ film was prepared by PLD, as described above. The surface exhibited steps ( $\sim 0.4 \mathrm{~nm})$ and terraces [Fig. 2(d)]. Then, 30-nm-thick Ti films were deposited with a stencil mask by electron beam (EB; base pressure $\sim 10^{-4}$, no substrate heating) evaporation, which was used as the source and drain electrodes. The channel length $L$ and the channel width $W$ of the TFT were 800 and $400 \mu \mathrm{m}$, respectively. Next, an $\sim 300$-nm-thick amorphous $12 \mathrm{CaO} \cdot 7 \mathrm{Al}_{2} \mathrm{O}_{3}\left(a-\mathrm{C} 12 \mathrm{~A} 7, \varepsilon_{r}=12\right)$ [20] film, which was used as a gate insulator, was deposited through a stencil mask by PLD. Finally, an 30-nm-thick Ti film was deposited by

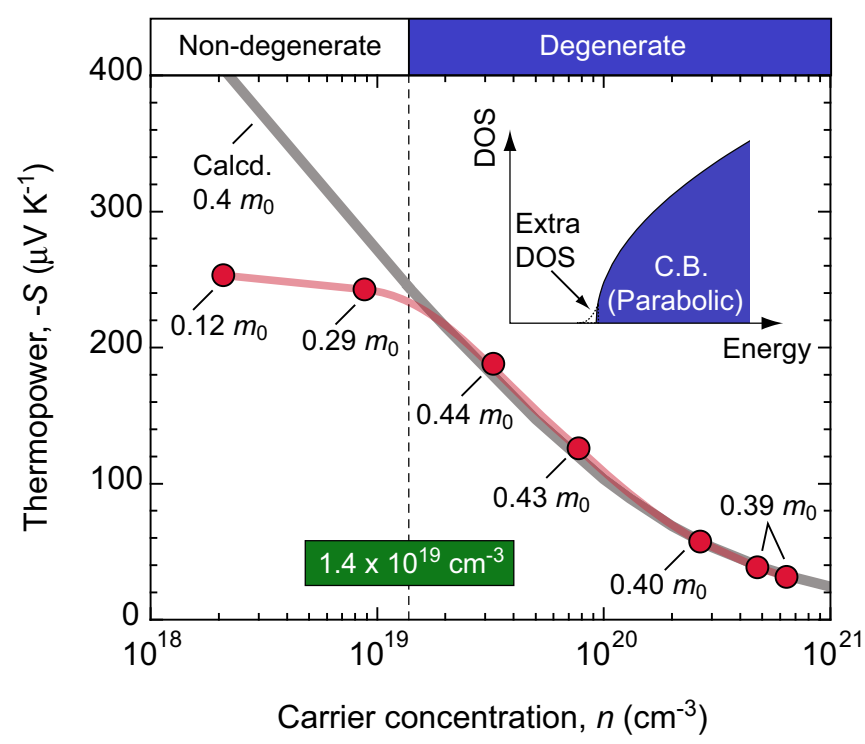

FIG. 1. Change in the thermopower $S$ of La-doped $\mathrm{BaSnO}_{3}$ films grown on (001) $\mathrm{SrTiO}_{3}$ substrates as a function of the carrier concentration $n$ at room temperature. The red line is to guide the eye. Effective mass $m^{*}$ values, which are calculated using Eqs. (1)-(3), are shown. The gray line is the $S-n$ curve calculated using $m^{*}=0.4 \pm 0.1 m_{0}$. The dotted line is the threshold of the degenerate/nondegenerate semiconductor around $n=1.4 \times 10^{19} \mathrm{~cm}^{-3}$. The inset shows a schematic explanation of the energy dependence of the DOS. 

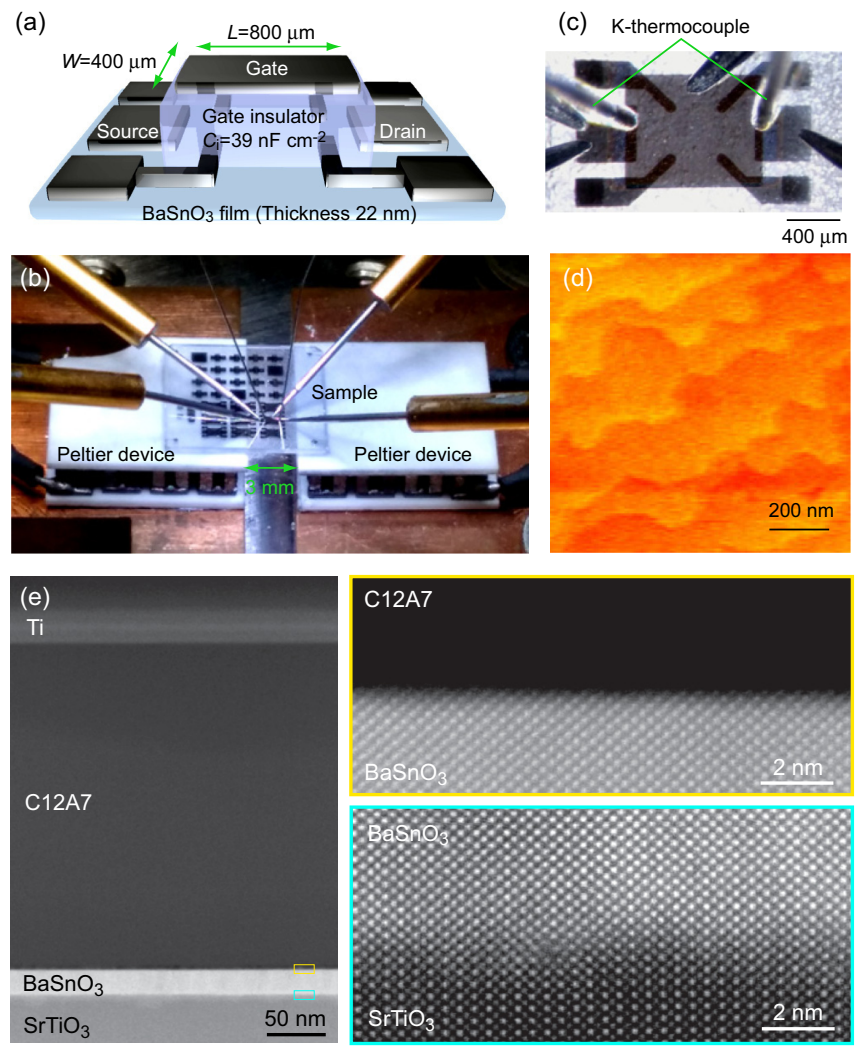

FIG. 2. $\mathrm{BaSnO}_{3}$ TFT structure [(a) schematic structure, (b) photograph of the thermopower measurement, (c) the magnified photograph, (d) topographic AFM image of the $\mathrm{BaSnO}_{3}$ film surface, and (e) cross-sectional HAADF-STEM images]. (a)-(c) Channel length $L$ and channel width $W$ of the TFT are 800 and $400 \mu \mathrm{m}$, respectively. Capacitance per unit area $C_{\mathrm{i}}$ of the $a-\mathrm{C} 12 \mathrm{~A} 7$ gate insulator is $39 \mathrm{nF} \mathrm{cm}^{-2}$. Two thermocouples (K type, $150 \mu \mathrm{m}$ in diameter, SHINNETSU), which are mechanically attached at both edges of the channel, monitor the temperature difference. (d) Stepped and terraced surface. (e) Multilayer structure of $\mathrm{Ti} / a-\mathrm{C} 12 \mathrm{~A} 7 / \mathrm{BaSnO}_{3} / \mathrm{SrTiO}_{3}$. $\mathrm{BaSnO}_{3}$ is heteroepitaxially grown on the (001) $\mathrm{SrTiO}_{3}$ substrate. The abrupt heterointerface between $a-\mathrm{C} 12 \mathrm{~A} 7 / \mathrm{BaSnO}_{3}$ is clearly shown.

EB evaporation as the top gate electrode. The as-fabricated TFT device was annealed at $150^{\circ} \mathrm{C}$ in air to reduce the off current. Details of our TFT fabrication process are described elsewhere [20-24].

Figure 2(e) shows the cross-sectional high angle annular dark field scanning transmission electron microscope (HAADF-STEM, JEM-ARM200F with an accelerating voltage of $200 \mathrm{kV}$ ) image of the $\mathrm{Ti} / a-\mathrm{C} 12 \mathrm{~A} 7 / \mathrm{BaSnO}_{3} / \mathrm{SrTiO}_{3}$ interfacial region. A multilayer structure and an abrupt heterointerface between $a-\mathrm{C} 12 \mathrm{~A} 7 / \mathrm{BaSnO}_{3}$ were clearly observed. The thicknesses of $\mathrm{Ti}, a-\mathrm{C} 12 \mathrm{~A} 7$, and $\mathrm{BaSnO}_{3}$ were 28,280 , and $22 \mathrm{~nm}$, respectively.

A semiconductor device analyzer (Agilent B1500A) was used to measure the transistor characteristics of the undoped $\mathrm{BaSnO}_{3}$ TFT at room temperature. Figure 3 summarizes the typical transistor characteristics such as the transfer and output characteristics, threshold voltage, and field effect mobility of the resultant $\mathrm{BaSnO}_{3}$ TFT. The drain current $I_{\mathrm{d}}$
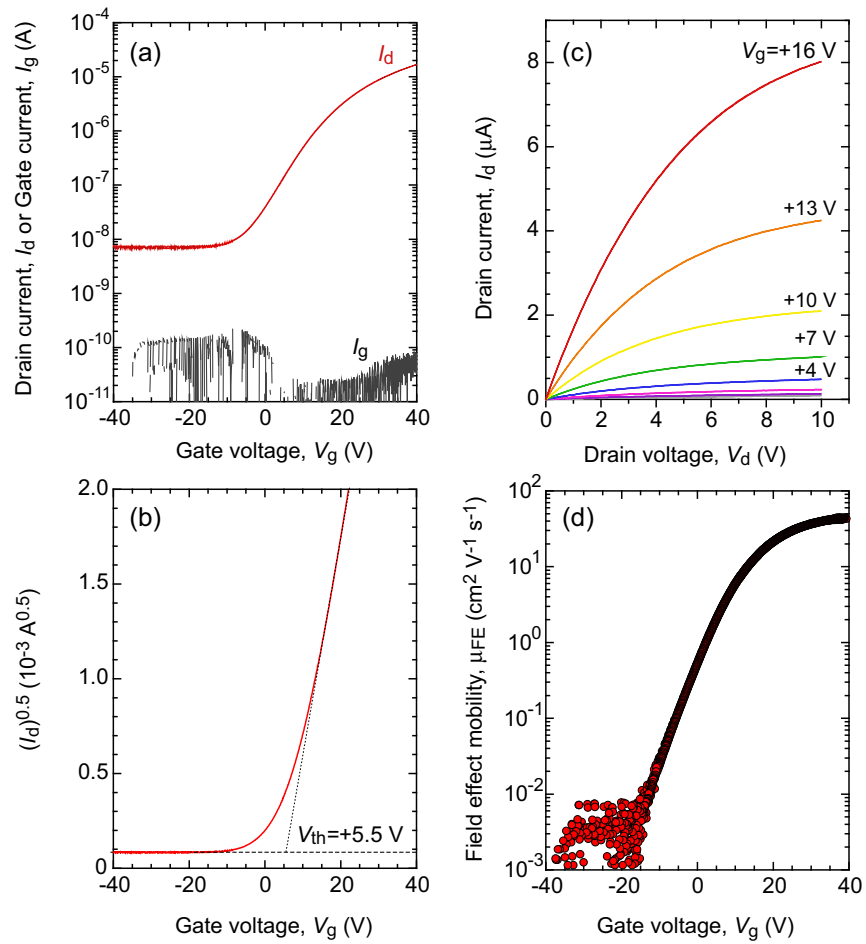

FIG. 3. Typical transistor characteristics of the resultant $\mathrm{BaSnO}_{3}$ TFT at room temperature [(a) transfer characteristics $I_{\mathrm{d}}-V_{\mathrm{g}}$ at $V_{\mathrm{d}}=$ $1 \mathrm{~V}$, (b) $I_{\mathrm{d}}^{0.5}-V_{\mathrm{g}}$ plot, (c) output characteristics $I_{\mathrm{d}}-V_{\mathrm{d}}$, and (d) field effect mobility $\left.\mu_{\text {Hall }}\right]$. The on-off current ratio is $\sim 10^{3}$. The threshold voltage $V_{\mathrm{th}}$ is $+5.5 \mathrm{~V}$. The maximum field effect mobility $\mu_{\mathrm{FE}}$ is $\sim 40 \mathrm{~cm}^{2} \mathrm{~V}^{-1} \mathrm{~s}^{-1}$.

increased markedly as the gate voltage $V_{\mathrm{g}}$ increased [Fig. 3(a)], indicating that the channel was $n$ type and the electron carriers accumulated by a positive $V_{\mathrm{g}}$. An on-off current ratio of $\sim 10^{3}$ was obtained for $V_{\mathrm{d}}=1 \mathrm{~V}$. A rather large threshold voltage $\left(V_{\mathrm{th}}\right)$ of $+5.5 \mathrm{~V}$ was observed from the linear fit of the $I_{\mathrm{d}}^{0.5}-V_{\mathrm{g}}$ plot [Fig. 3(b)]. A clear pinch-off behavior and current saturation in $I_{\mathrm{d}}$ revealed that the resultant TFT obeyed the standard field effect transistor theory [Fig. 3(c)]. The field effect mobility $\mu_{\mathrm{FE}}$ was calculated from $\mu_{\mathrm{FE}}=$ $g_{\mathrm{m}}\left[(W / L) C_{\mathrm{i}} V_{\mathrm{d}}\right]^{-1}$, where $g_{\mathrm{m}}$ is the transconductance $\partial I_{\mathrm{d}} / \partial V_{\mathrm{g}}$ and $C_{\mathrm{i}}$ is the capacitance per unit area $\left(39 \mathrm{nF} \mathrm{cm}^{-2}\right)$ [Fig. 3(d)]. $\mu_{\mathrm{FE}}$ drastically increased with $V_{\mathrm{g}}$ and became saturated at $\sim 40 \mathrm{~cm}^{2} \mathrm{~V}^{-1} \mathrm{~s}^{-1}$, which was $\sim 60 \%$ of the room temperature $\mu_{\text {Hall }}$ of La-doped $\mathrm{BaSnO}_{3}\left(\mu_{\text {Hall }} \sim 67 \mathrm{~cm}^{2} \mathrm{~V}^{-1} \mathrm{~s}^{-1}\right)$.

Then, we measured the electric field modulated $S$ of the resultant $\mathrm{BaSnO}_{3}$ TFT (Fig. 2). For the $S$ measurements, we used two Peltier devices, which were placed under the TFT, to generate a temperature difference between the source and drain electrodes [Fig. 2(b)]. Two thermocouples (K type, $150 \mu \mathrm{m}$ in diameter, SHINNETSU), which were mechanically attached at both edges of the channel, monitored the temperature difference $\Delta T(0-5 \mathrm{~K})$ [Fig. 2(c)]. The thermoelectromotive force $\Delta V$ and $\Delta T$ values were simultaneously measured at room temperature. The $S$ values were obtained from the slope of the $\Delta V-\Delta T$ plots. Details of our electric field modulated $S$ measurements are described elsewhere [20,21,23-26]. 


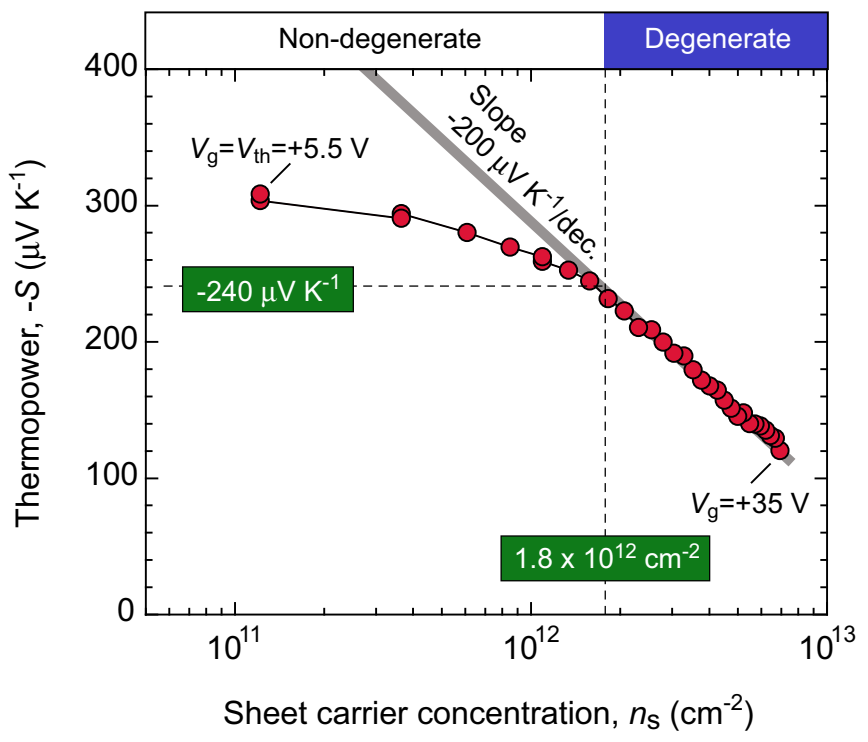

FIG. 4. Electric field modulated $S$ of the resultant $\mathrm{BaSnO}_{3}$ TFT as a function of the sheet carrier concentration at room temperature. The inflection point occurs around $\left(|S|, n_{s}\right)=\left(240 \mu \mathrm{V} \mathrm{K} K^{-1}, 1.8 \times\right.$ $\left.10^{12} \mathrm{~cm}^{-2}\right)$. The slope of the $S-\log n_{\mathrm{s}}$ plot is $\sim 200 \mu \mathrm{V} \mathrm{K}^{-1} /$ decade (gray line) when $n_{\mathrm{s}}$ exceeds $1.8 \times 10^{12} \mathrm{~cm}^{-2}$. These data indicate the threshold of a degenerate/nondegenerate semiconductor is around $n=1.8 \times 10^{12} \mathrm{~cm}^{-2}$.

Figure 4 shows the electric field modulated $S$ of the resultant $\mathrm{BaSnO}_{3}$ TFT as a function of sheet carrier concentration $n_{\mathrm{s}}$, which was deduced from $n_{\mathrm{s}}=C_{\mathrm{i}}\left(V_{\mathrm{g}}-V_{\mathrm{th}}\right)$. The $|S|$ value gradually decreased from 308 to $120 \mu \mathrm{V} \mathrm{K}^{-1}$ with $n_{\mathrm{s}}$, which is consistent with the La-doped $\mathrm{BaSnO}_{3}$ films (Fig. 1). It should be noted that a deflection point occurred around $\left(|S|, n_{s}\right)=\left(240 \mu \mathrm{V} \mathrm{K} K^{-1}, 1.8 \times 10^{12} \mathrm{~cm}^{-2}\right)$. An almost linear relationship with a slope with of $\sim 200 \mu \mathrm{V} \mathrm{K}^{-1} /$ decade was observed in the $S-\log n_{\mathrm{s}}$ plot when $n_{\mathrm{s}}$ exceeded $1.8 \times$ $10^{12} \mathrm{~cm}^{-2}$. Below $1.8 \times 10^{12} \mathrm{~cm}^{-2}$, the slope was not linear. This observation is similar to that of the La-doped $\mathrm{BaSnO}_{3}$ films; the degenerate/nondegenerate threshold was around $\left(|S|, n_{s}\right)=\left(240 \mu \mathrm{V} \mathrm{K} K^{-1}, 1.8 \times 10^{12} \mathrm{~cm}^{-2}\right)$. In the degenerate region, the $E_{\mathrm{F}}$ was located above the threshold and $S$ obeyed Eqs. (1)-(3). The conduction band bottom was parabolic shaped and the intrinsic $m^{*}$ was $0.40 \pm 0.01 m_{0}$.

In the nondegenerate region, $E_{\mathrm{F}}$ was below the threshold. The $S$ value did not show a clear $n$ dependence. Similar phenomena were also observed in amorphous TOSs, $a-\mathrm{InGaZnO}_{4}$, and $a-\mathrm{In}_{2} \mathrm{MgO}_{4}$ [22]. In the case of these amorphous TOSs, structural imperfections led to the formation of an antiparabolic shaped extra state just below the original conduction band bottom, which played an essential role in the transistor switching of TOS-based TFTs [27,28]. In the present case, the almost linear shaped extra DOS (i.e., tail states) formed just below the conduction band bottom, possibly due to an oxygen deficiency. Because these tail states suppressed the carrier mobility, $\mathrm{BaSnO}_{3}$ films exhibited a very low mobility when $E_{\mathrm{F}}$ was below the threshold.

In summary, we have clarified the intrinsic effective mass of a transparent oxide semiconductor $\mathrm{BaSnO}_{3}, m^{*}=$ $0.40 \pm 0.01 m_{0}$, using the thermopower modulation clarification method. We also determined the threshold of the degenerate/nondegenerate semiconductor of $\mathrm{BaSnO}_{3}$. At the threshold, both La-doped $\mathrm{BaSnO}_{3}$ and the $\mathrm{BaSnO}_{3}$ TFT structures have a thermopower value of $240 \mu \mathrm{V} \mathrm{K}^{-1}$, a bulk carrier concentration of $1.4 \times 10^{19} \mathrm{~cm}^{-3}$, and a two-dimensional sheet carrier concentration of $1.8 \times 10^{12} \mathrm{~cm}^{-2}$. A rather high mobility occurs when $E_{\mathrm{F}}$ is located above the parabolic shaped conduction band bottom. On the contrary, a very low carrier mobility is observed when the $E_{\mathrm{F}}$ is below the threshold, most likely because the tail states suppress the carrier mobility.

We hope the present results will lead to further developments of $\mathrm{BaSnO}_{3}$-based oxide electronics.

This research was supported by Grants-in-Aid for Scientific Research on Innovative Areas "Nano Informatics" (25106003 and 25106007) from the Japan Society for the Promotion of Science (JSPS). H.O. was supported by Grants-in-Aid for Scientific Research A (17H01314) and B (26287064) from the JSPS and the Asahi Glass Foundation. Part of this work was supported by "Nanotechnology Platform" (12024046), sponsored by MEXT, Japan.
[1] Handbook of Transparent Conductors, edited by D. S. McGinley (Springer, Berlin, 2010).

[2] H. J. Kim, U. Kim, H. M. Kim, T. H. Kim, H. S. Mun, B. G. Jeon, K. T. Hong, W. J. Lee, C. Ju, K. H. Kim, and K. Char, High mobility in a stable transparent perovskite oxide, Appl. Phys. Express. 5, 061102 (2012).

[3] H. J. Kim, U. Kim, T. H. Kim, J. Kim, H. M. Kim, B. G. Jeon, W. J. Lee, H. S. Mun, K. T. Hong, J. Yu, K. Char, and K. H. Kim, Physical properties of transparent perovskite oxides $(\mathrm{Ba}, \mathrm{La}) \mathrm{SnO}_{3}$ with high electrical mobility at room temperature, Phys. Rev. B 86, 165205 (2012).

[4] P. V. Wadekar, J. Alaria, M. O'Sullivan, N. L. O. Flack, T. D. Manning, L. J. Phillips, K. Durose, O. Lozano, S. Lucas, J. B. Claridge, and M. J. Rosseinsky, Improved electrical mobility in highly epitaxial $\mathrm{La}: \mathrm{BaSnO}_{3}$ films on $\mathrm{SmScO}_{3}$ (110) substrates, Appl. Phys. Lett. 105, 052104 (2014).
[5] C. Park, U. Kim, C. J. Ju, J. S. Park, Y. M. Kim, and K. Char, High mobility field effect transistor based on $\mathrm{BaSnO}_{3}$ with $\mathrm{Al}_{2} \mathrm{O}_{3}$ gate oxide, Appl. Phys. Lett. 105, 203503 (2014).

[6] U. Kim, C. Park, T. Ha, Y. M. Kim, N. Kim, C. Ju, J. Park, J. Yu, J. H. Kim, and K. Char, All-perovskite transparent high mobility field effect using epitaxial $\mathrm{BaSnO}_{3}$ and $\mathrm{LaInO}_{3}$, APL Mater. 3, 036101 (2015).

[7] S. Raghavan, T. Schumann, H. Kim, J. Y. Zhang, T. A. Cain, and S. Stemmer, High-mobility $\mathrm{BaSnO}_{3}$ grown by oxide molecular beam epitaxy, APL Mater. 4, 016106 (2016).

[8] S. J. Allen, S. Raghavan, T. Schumann, K. M. Law, and S. Stemmer, Conduction band edge effective mass of La-doped $\mathrm{BaSnO}_{3}$, Appl. Phys. Lett. 108, 252107 (2016).

[9] J. Shin, Y. M. Kim, Y. Kim, C. Park, and K. Char, High mobility $\mathrm{BaSnO}_{3}$ films and field effect transistors on non-perovskite $\mathrm{MgO}$ substrate, Appl. Phys. Lett. 109, 262102 (2016). 
[10] C. A. Niedermeier, S. Rhode, S. Fearn, K. Ide, M. A. Moram, H. Hiramatsu, H. Hosono, and T. Kamiya, Solid phase epitaxial growth of high mobility $\mathrm{La}: \mathrm{BaSnO}_{3}$ thin films co-doped with interstitial hydrogen, Appl. Phys. Lett. 108, 172101 (2016).

[11] E. Moreira, J. M. Henriques, D. L. Azevedo, E. W. S. Caetano, V. N. Freire, U. L. Fulco, and E. L. Albuquerque, Structural and optoelectronic properties, and infrared spectrum of cubic $\mathrm{BaSnO}_{3}$ from first principles calculations, J. Appl. Phys. 112, 043703 (2012).

[12] H. R. Liu, J. H. Yang, H. J. Xiang, X. G. Gong, and S. H. Wei, Origin of the superior conductivity of perovskite $\mathrm{Ba}(\mathrm{Sr}) \mathrm{SnO}_{3}$, Appl. Phys. Lett. 102, 112109 (2013)

[13] B. Hadjarab, A. Bouguelia, and M. Trari, Optical and transport properties of lanthanum-doped stannate $\mathrm{BaSnO}_{3}$, J. Phys. D: Appl. Phys. 40, 5833 (2007).

[14] H. J. Kim, J. Kim, T. H. Kim, W. J. Lee, B. G. Jeon, J. Y. Park, W. S. Choi, D. W. Jeong, S. H. Lee, J. Yu, T. W. Noh, and K. H. Kim, Indications of strong neutral impurity scattering in $\mathrm{Ba}(\mathrm{Sn}, \mathrm{Sb}) \mathrm{O}_{3}$ single crystals, Phys. Rev. B 88, 125204 (2013).

[15] D. Seo, K. Yu, Y. J. Chang, E. Sohn, K. H. Kim, and E. J. Choi, Infrared-optical spectroscopy of transparent conducting perovskite ( $\mathrm{La}, \mathrm{Ba}) \mathrm{SnO}_{3}$ thin films, Appl. Phys. Lett. 104, 022102 (2014).

[16] B. C. Luo, X. S. Cao, K. X. Jin, and C. L. Chen, Determination of the effective mass and nanoscale electrical transport in La-doped $\mathrm{BaSnO}_{3}$ thin films, Curr. Appl. Phys. 16, 20 (2016).

[17] J. Shiogai, K. Nishihara, K. Sato, and A. Tsukazaki, Improvement of electron mobility in $\mathrm{La}: \mathrm{BaSnO}_{3}$ thin films by insertion of an atomically flat insulating $(\mathrm{Sr}, \mathrm{Ba}) \mathrm{SnO}_{3}$ buffer layer, AIP Adv. 6, 065305 (2016).

[18] See Supplemental Material at http://link.aps.org/supplemental/ 10.1103/PhysRevMaterials.1.034603 for temperature dependence of electrical resistivity of the La-doped $\mathrm{BaSnO}_{3}$ films with varied carrier concentrations, out-of-plane XRD pattern, $\mathrm{x}$-ray rocking curve (XRC), and x-ray reflectivity curve (XRR) of the resultant $\mathrm{BaSnO}_{3}$ film.
[19] C. B. Vining, A model for the high-temperature transport properties of heavily doped $n$-type silicon-germanium alloys, J. Appl. Phys. 69, 331 (1991).

[20] H. Ohta, Y. Masuoka, R. Asahi, T. Kato, Y. Ikuhara, K. Nomura, and $\mathrm{H}$. Hosono, Field-modulated thermopower in $\mathrm{SrTiO}_{3}$-based field-effect transistors with amorphous $12 \mathrm{CaO} \cdot 7 \mathrm{Al}_{2} \mathrm{O}_{3}$ glass gate insulator, Appl. Phys. Lett. 95, 113505 (2009).

[21] A. Yoshikawa, K. Uchida, K. Koumoto, T. Kato, Y. Ikuhara, and $\mathrm{H}$. Ohta, Electric-field modulation of thermopower for the $\mathrm{KTaO}_{3}$ field-effect transistors, Appl. Phys. Express 2, 121103 (2009).

[22] K. Uchida, A. Yoshikawa, K. Koumoto, T. Kato, Y. Ikuhara, and H. Ohta, A single crystalline strontium titanate thin film transistor, J. Appl. Phys. 107, 096103 (2010).

[23] H. Koide, Y. Nagao, K. Koumoto, Y. Takasaki, T. Umemura, T. Kato, Y. Ikuhara, and H. Ohta, Electric field modulation of thermopower for transparent amorphous oxide thin film transistors, Appl. Phys. Lett. 97, 182105 (2010).

[24] Y. Nagao, A. Yoshikawa, K. Koumoto, T. Kato, Y. Ikuhara, and $\mathrm{H}$. Ohta, Experimental characterization of the electronic structure of anatase $\mathrm{TiO}_{2}$ : Thermopower modulation, Appl. Phys. Lett. 97, 172112 (2010).

[25] H. Ohta, T. Mizuno, S. J. Zheng, T. Kato, Y. Ikuhara, K. Abe, H. Kumomi, K. Nomura, and H. Hosono, Unusually large enhancement of thermopower in an electric field induced two-dimensional electron gas, Adv. Mater. 24, 740 (2012).

[26] H. Ohta, Y. Sato, T. Kato, S. Kim, K. Nomura, Y. Ikuhara, and H. Hosono, Field-induced water electrolysis switches an oxide semiconductor from an insulator to a metal, Nat. Commun. 1, 118 (2010).

[27] K. Nomura, T. Kamiya, H. Yanagi, E. Ikenaga, K. Yang, K. Kobayashi, M. Hirano, and H. Hosono, Subgap states in transparent amorphous oxide semiconductor, In-Ga-Zn-O, observed by bulk sensitive x-ray photoelectron spectroscopy, Appl. Phys. Lett. 92, 202117 (2008).

[28] W. Korner, D. F. Urban, and C. Elsasser, Generic origin of subgap states in transparent amorphous semiconductor oxides illustrated for the cases of In-Zn-O and In-Sn-O, Phys. Status Solidi A 212, 1476 (2015). 